\title{
THE HEMODYNAMIC ALTERATIONS PRODUCED BY A PLASTIC VALVULAR PROSTHESIS FOR SEVERE AORTIC INSUFFICIENCY IN MAN 1, 2
}

\author{
By JOHN C. ROSE, CHARLES A. HUFNAGEL, EDWARD D. FREIS, W. PROCTOR \\ HARVEY, AND EDWARD A. PARTENOPE \\ (From The Departments of Medicine and Surgery, Georgetown University School of Medicine, \\ The Cardiovascular Research Laboratory, Georgetown University Hospital \\ and the Veterans Administration Hospital, Washington, D. C.)
}

(Submitted for publication November 12, 1953; accepted February 18, 1954)

Until recently, patients with aortic insufficiency and congestive heart failure have been offered only the temporary palliation provided by rest, diet, cardiac glycosides, and diuretics. A more direct therapeutic approach would aim at correction of the basic hemodynamic defect, by mechanical reduction of the volume of regurgitation.

Hufnagel (1) has devised a surgical procedure for altering the abnormal pattern of blood flow in aortic insufficiency. A plastic ball valve is inserted in the thoracic aorta, immediately distal to the left subclavian artèry. This prosthesis prevents regurgitation of the blood traversing it, estimated to be approximately 75 per cent of the total cardiac output (2) (Figure 1). Blood flow above the valve, in the coronary, carotid, and subclavian systems remains regurgitant.

To date, over twenty-five patients have been treated successfully in this manner $(3,4)$. Clinical observations suggest that patients with severe aortic insufficiency and varying degrees of cardiac decompensation benefit markedly by this operation, showing postoperatively increased exercise tolerance and clearing of the signs of heart failure. The present study was undertaken in order to demonstrate objectively the alterations in circulatory dynamics produced by the valvular prosthesis.

\section{MATERIAL AND METHODS}

Nine patients (see Appendix) with severe aortic insufficiency as an isolated valve lesion were studied pre-

1 Presented in part at the Forty-fifth Annual Meeting of the American Society for Clinical Investigation, Atlantic City, New Jersey, May 4, 1953.

2 Sponsored in part by the Veterans Administration and published with the approval of the Chief Medical Director. The statements and conclusions published by the authors are a result of their own study and do not necessarily reflect the opinion or policy of the Veterans Administration. operatively and from 14 to 36 days postoperatively. All preoperative studies were carried out after several weeks of medical treatment in the hospital. The procedure of study was as follows:

Each patient fasted from midnight. Approximately 20 minutes before being brought to the laboratory they received $200 \mathrm{mg}$. sodium pentobarbital (Nembutal) orally.

Using local procaine anesthesia, a Cournand catheter was passed to the right heart under direct fluoroscopic control. The zero level for all right heart pressures was $5 \mathrm{~cm}$. posterior to the angle of Louis. After recording the pulmonary arterial pressure, the catheter was withdrawn to the cavity of the right ventricle and left in place. In one patient (L. F.), the catheter was withdrawn to the right auricle because of the occurrence of premature ventricular contractions. This was the only case in which an ectopic rhythm was observed. In another ( $\mathrm{H}$. S.), the catheter could not be passed to the right heart; hence, direct Fick cardiac outputs were not obtained in this case.

A 15 gauge Robb type needle $5 \mathrm{~cm}$. in length (5) was inserted through procainized skin into the femoral artery. The ample bore of this needle permitted unrestricted flow and adequate sampling when obtaining dye concentration curves.

Cardiac output was determined by the direct Fick method. Inspired air volume was measured from a Tissot spirometer (filled with room air) over a threeminute period. Expired air samples were collected in Douglas bags and their oxygen content determined immediately by a Pauling oxygen analyzer. ${ }^{3}$ Oxygen consumption was calculated from expired air oxygen concentration, room air oxygen concentration, and inspired air volume corrected for temperature and barometric pressure. During the second minute of the three-minute air sampling period, simultaneous blood samples were drawn slowly from the right heart and femoral artery into oiled syringes. These were capped with mercury, kept on ice, and within three hours were analyzed for arteriovenous oxygen differences in a quartz spectrophotometer ${ }^{4}$ by the method of Hickam and Frayser (6).

\footnotetext{
3 Arnold O. Beckman Company, Inc., South Pasadena, California.

4 Arnold O. Beckman Company, Inc., South Pasadena, California.
} 


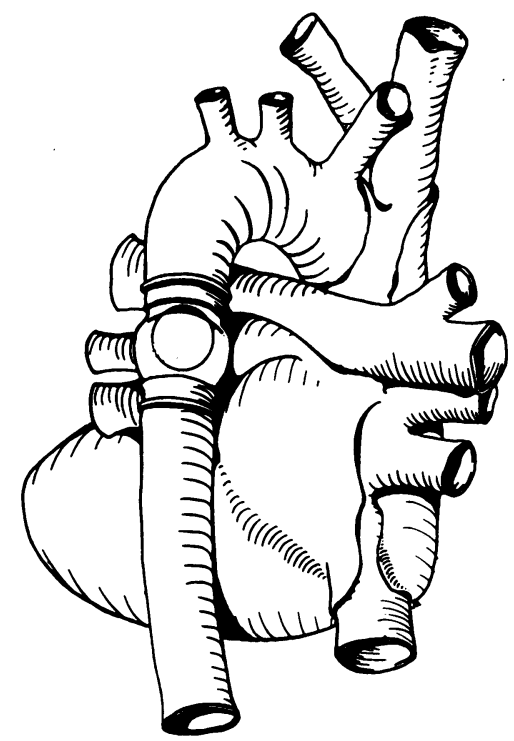

Fig. 1. Sketch of the Posterior Aspect of the Heart and Great Vessels, Showing the Location of the Plastic Aortic Valve in the Thoracic Aorta, beyond the Left Subclavian Artery

An 18 gauge needle was then inserted in the brachial artery under local procaine anesthesia, and direct arterial pressure pulse contours were obtained. All pressures were recorded with strain gage manometers ${ }^{5}$ using carrier-wave type strain gage amplifiers. ${ }^{6}$ The strain gages were coupled directly to the large bore indwelling needles in both femoral and brachial arteries, with no in-

5 Statham Laboratories, Beverly Hills, California.

6 Sanborn Company, Cambridge, Massachusetts. tervening tubing. The frequency response curve of the direct-writing recorders used is flat to fifty cycles per second, and a 60 per cent response is obtained at $80 \mathrm{cy}$ cles per second. (In obtaining right heart pressures, a strain-gage was coupled directly to the hub of the indwelling catheter.)

The injection of T-1824 dye into the right heart, and arterial sampling were modified from the method of Werkö and his associates (7). The precise amount of dye delivered through each catheter into the right ventricle was determined by an in vitro injection. In every case, the same catheter used in the preoperative study was used in the postoperative study, and in each instance the approximate position of the catheter in the right heart was duplicated in the postoperative procedure. All blood samples were collected in paraffined tubes each containing a drop of one per cent heparin. In four cases, during the postoperative study, dye concentration curves were obtained from the brachial artery simultaneously with the femoral dye curves. The mean circulation times were obtained from the dye concentration curves by the method of Hamilton, Moore, Kinsman, and Spurling (8).

After obtaining the dye curve, additional arterial samples were obtained at 10,15 , and 20 minutes after the injection of dye, for determination of plasma volume (9). Total blood volume was calculated from the plasma volume and arterial hematocrit.

\section{RESULTS}

\section{Brachial and femoral arterial pressure pulse contours}

Tracings taken from the femoral artery postoperatively supplied evidence that the valve was
BEFORE SURGERY

AFTER SURGERY

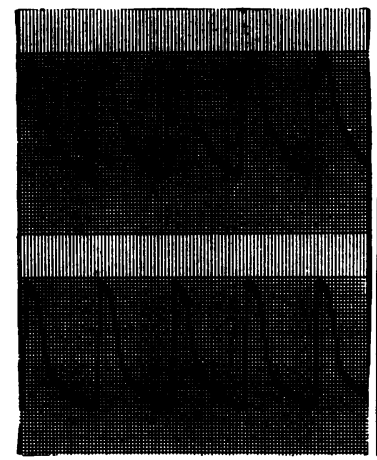

PATIENT: H.S.

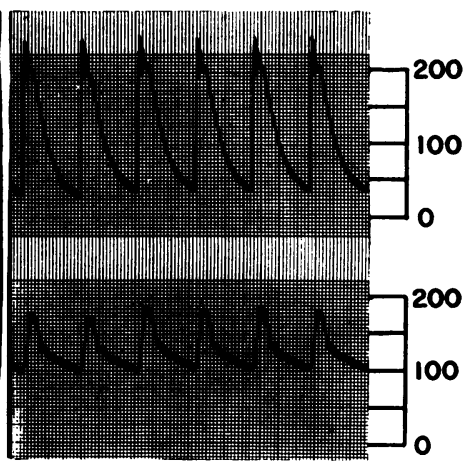

MM.HG.

Fig. 2. Cuttings Taken from Direct Recordings of Arterial Pressure Pulse Contours in the Brachial and Femoral Artery in Patient H. S. Before and after Surgery

While the wide regurgitant pressure pulse is transformed into a normal appearing contour in the femoral artery postoperatively, the brachial contour is widened due to an increased systolic pressure and a decreased diastolic pressure. 
BRACHIAL ARTERIAL PRESSURE, MM.HG.
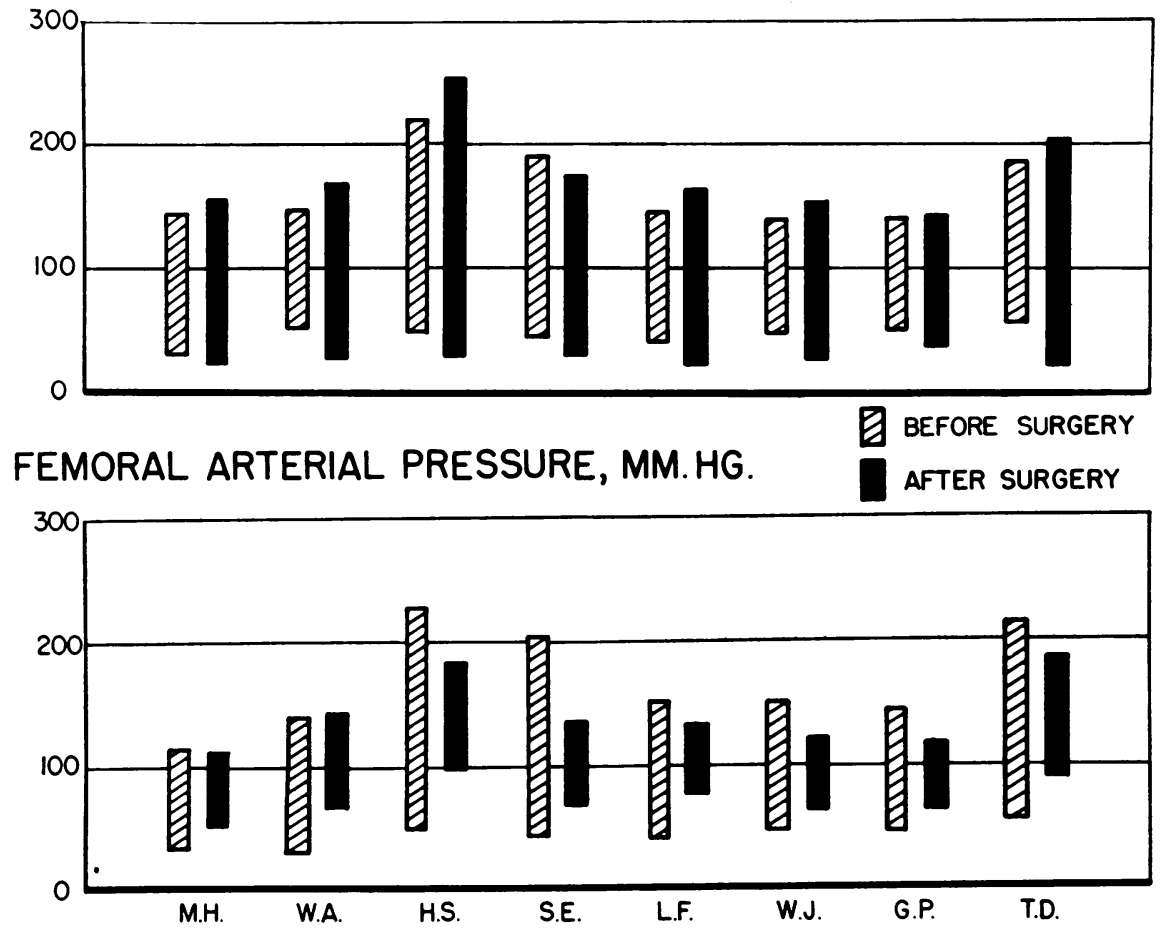

Fig. 3. Chart Summarizing the Arterial Pressure Changes Produced by the Plastic Valve in Eight Cases

In each instance the femoral pressures are altered towards normal, while the pulse pressure in the brachial artery is widened. In patient M. H., the preoperative brachial pulse pressure is slightly wider than the preoperative femoral pulse pressure. At operation a slightly coarcted area was found in the descending thoracic aorta which did not interfere with normal aortic blood flow. No collateral channels had developed, and this segment was left intact, the prosthesis being placed proximal to it.

functioning effectively. In each instance, the pressure pulse contour characteristic of free aortic regurgitation before operation was replaced after operation by a contour which appeared normal (Figures 2 and 3). Whereas the femoral arterial pulse pressure decreased toward normal, the brachial arterial pulse pressure widened postoperatively, due to both a decrease in diastolic pressure and (with one exception, S. E.) an increase in systolic pressure.

The configuration of the postoperative femoral arterial pressure pulse was of interest in that in most cases, a peculiar and variable notching occurred, perhaps due to closure of the rigid ball valve (Figure 4).

\section{Cardiac output and stroke volume}

In six cases, the cardiac index increased postoperatively by from 19 to 77 per cent of the preop- erative values (Table I). The two exceptions were S. E., who exhibited no significant change, and T. D., who showed a decrease of 34 per cent in cardiac output. In S. E., the preoperative values may have been falsely high because of evident apprehension during the experimental procedure. For a similar reason the preoperative cardiac output in patient T. D., may not represent a basal value. His cardiac index preoperatively was the highest recorded in the eight cases studied, and the difference in the pre and postoperative cardiac output determinations lay entirely in the oxygen consumption, the arteriovenous oxygen difference actually decreasing postoperatively.

An increase in cardiac output usually was associated with both increase in oxygen consumption and a decrease in $\mathrm{A}-\mathrm{V}$ oxygen difference. The exception to this was L. F., who exhibited a fall in oxygen consumption postoperatively, al- 
J. C. ROSE, C. A. HUfNAgel, E. D. FReis, W. P. haRvey, AND E. A. PARTENOpe

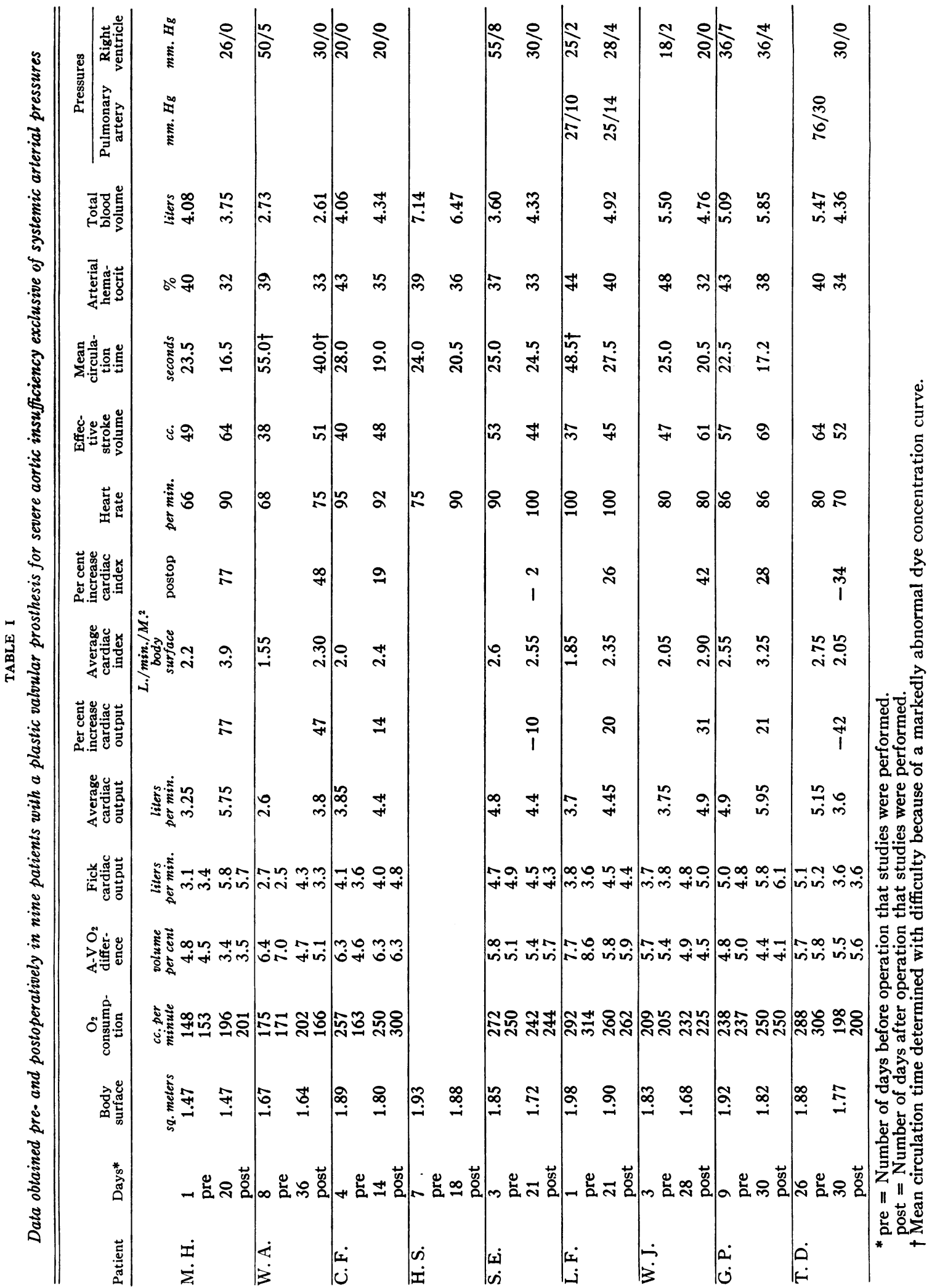




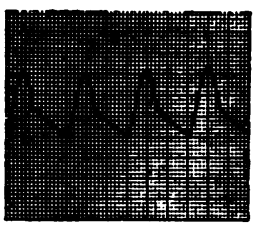

M.H.

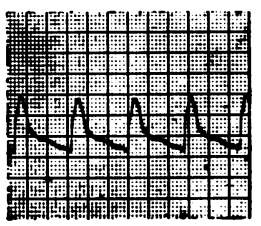

S.E.

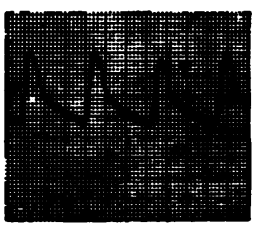

H.S.

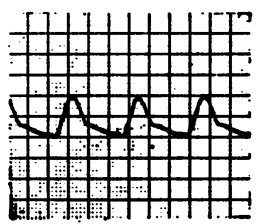

G.P.

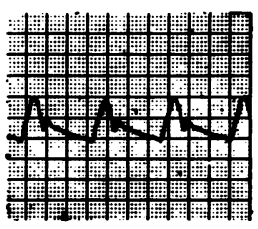

W.J.

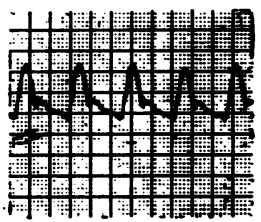

LF.

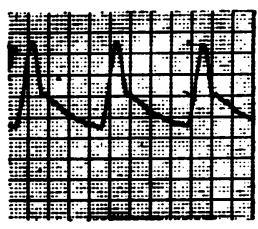

W.A.

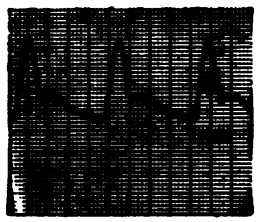

T.D.

Fig. 4. Cuttings from the Postoperative Femoral. Arterial. Pressure Puise Tracings in Eight Patients

Although the general configuration of the pressure pulse is normal, there is a variable notching which is presumably due to closure of the rigid ball valve in the thoracic aorta.

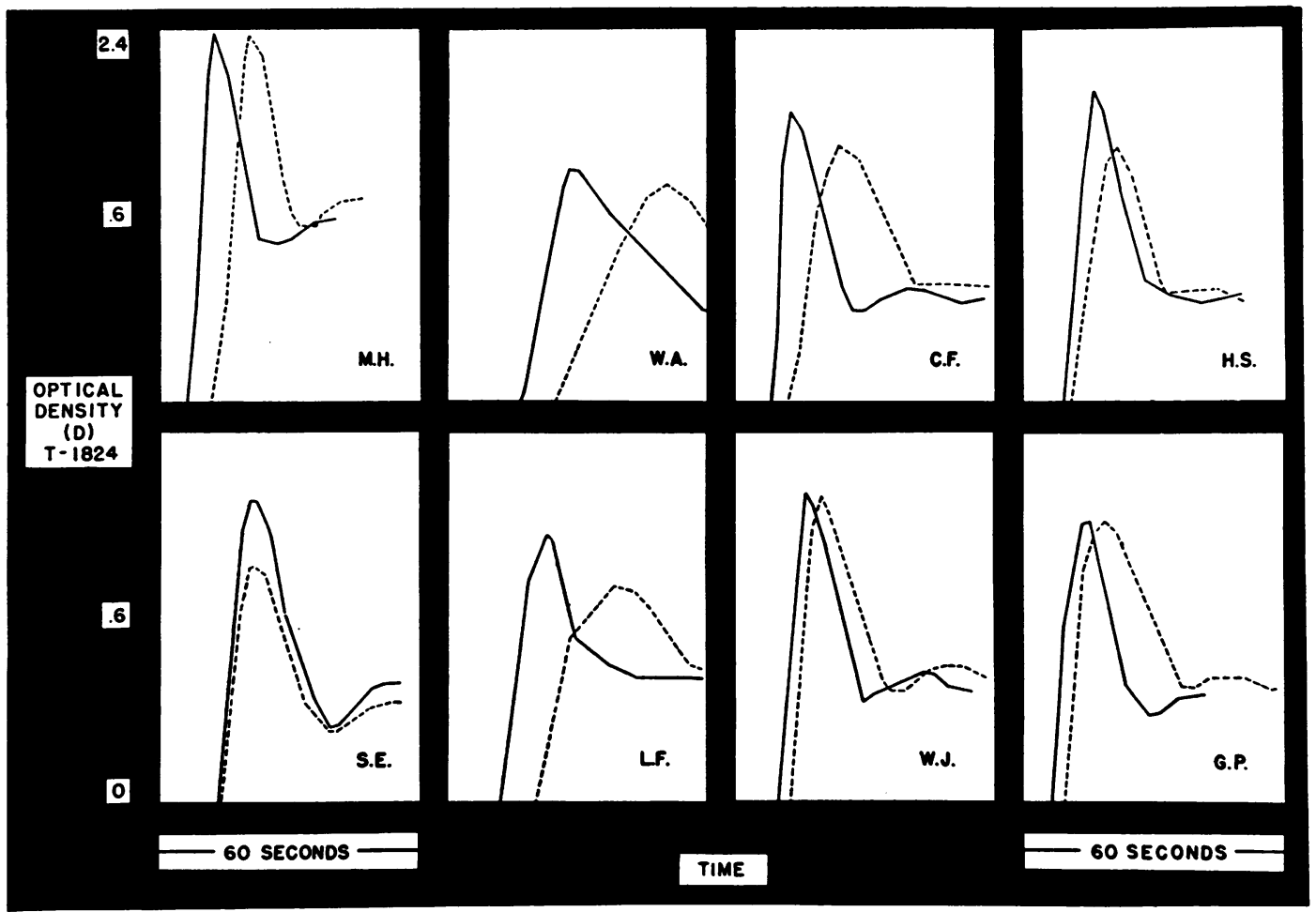

Fig. 5. Pre- and Postopierative Femoral T-1824 Dye Concentration Curves in Eight Pat ients

The preoperative curves are the broken lines, the postoperative curves the solid lines. Each abscissa is a linear time scale from 0 to 60 seconds. Each ordinate is a logarithmic optical density scale from 0 to 2.4. With the exception of patient S. E.; the postoperative femoral dye curve is steeper and more rapid than the preoperative curve. See text for discussion. 
though there was a greater proportionate fall in A-V oxygen difference. In patient $W$. A., one of the increased postoperative cardiac outputs was due entirely to a decrease in $\mathrm{A}-\mathrm{V}$ oxygen difference.

The effective stroke volume, calculated by dividing the average cardiac output by the heart rate recorded at the actual time of cardiac output measurement, usually increased paralleling the changes which occurred in cardiac output.

Certain discrepancies in the data were evident. One preoperative cardiac output value in patient C. F. was greater than one postoperative letermination, although the average of the postoperative outputs was greater by 14 per cent. In all but one case, there was weight loss following operation. Weight loss was not due to diuresis, but rather the body tissue loss observed following major surgery, and in each instance had either ceased or reversed itself at the time of postoperative study. The decrease in body surface area postoperatively accounted for somewhat greater percentile increases in cardiac indices in the cases in which the cardiac output increased, and smaller percentile decreases in cardiac indices in the two cases in which the cardiac output decreased.

\section{Right heart pressures}

Of the seven cases in which right heart pressures were measured no change was observed in four while postoperative decreases were observed in three (W. A., S. E., and T. D., Table I). These were the only ones exhibiting elevations of right heart pressure preoperatively. In the case of T. D., the preoperative pulmonary artery pressure was $76 / 30 \mathrm{~mm}$. $\mathrm{Hg}$, and although the postoperative pressure was obtained in the right ventricle, it was significantly lower than the preoperative right heart pressure in the face of a marked decrease in postoperative cardiac output. S. E. showed no significant alteration in cardiac index. yet there was a postoperative fall in right heart pressure. These observations may be further evidence that the preoperative cardiac outputs in these two cases do not represent basal values.

\section{T-1824 dye concentration curves}

The alterations in shape of dye concentration curves obtained in the femoral artery in eight cases are shown in Figure 5. With one exception, the postoperative dye curve is steeper and more rapid than the preoperative curve. The exception is patient S. E.. the only patient of this group who did not show a postoperative increase in cardiac output, or a postoperative increase in brachial artery systolic pressure. Mean circulation times calculated from these curves are summarized in Table $I$. The greatest reductions in mean circulation time occurred in the patients who showed the most alnormal preoperative values (W. A.. L. F.).

In four cases postoperatively, dye transit curves were determined simultaneously in both the femoral and the brachial arteries (Figure 6). Despite the persistence of regurgitant flow above the valve. the alterations in the brachial dye curve

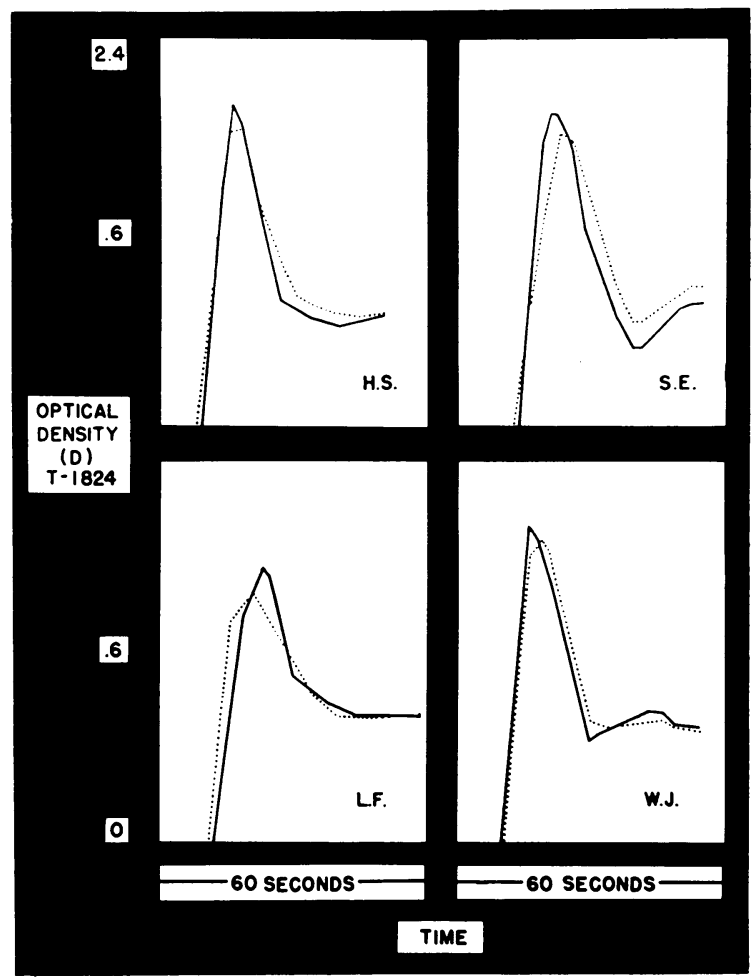

Fig. 6. Simultaneols Postoperative Dye Concextration Curves Drawix an Brachial axi Ffaroral. Arteries in Folk Patients

Brachial curves are the dotted lines, femoral curves the solid lines. Reference to Figure 5 reveals that in each instance the alterations which occur in the shape and duration of postoperative femoral dye curves are simultaneously noted in the brachial dye curves. See text for discussion. 
postoperatively corresponded to those in the femoral curve.

\section{Blood volume}

In all of the eight cases studied the arterial hematocrit was lower postoperatively. The reduction varied between 3 and $8 \mathrm{~mm}$., except for patient W. J., whose hematocrit fell $16 \mathrm{~mm}$. (Table I). Despite this constant finding there was no significant trend in total blood volumes, a slight postoperative rise occurring in three cases and a fall in five.

\section{DISCUSSION}

The cardiac output determinations and dye injection curves implied that the operation resulted in improved cardiac function. Cardiac output, cardiac index, and effective stroke volume were significantly increased in six of eight cases, postoperatively. Dye injection curves obtained from the brachial artery postoperatively showed a definite return toward normal in shape and duration corresponding to the alterations occurring in the femoral curves. Thus, the improvements in the femoral dye curves were not due to the correction of regurgitation, per se, since this improvement also was noted in the regurgitant brachial artery.

The source of improvement, therefore, seemed more likely to be cardiac in origin, possibly being secondary to more efficient ventricular contraction. This interpretation was supported by the cardiac output data. Those cases which exhibited an increase in cardiac output postoperatively also showed dye concentration curves of shorter duration, while the one patient with no change in cardiac output studied with dye injections failed to exhibit this dye curve alteration.

Considering the data as a whole, the following sequence of events seems to explain best the resuilts observed: the introduction of the plastic valve reduced the absolute volume of regurgitation. Ventricular contraction then became more effective, permitting better emptying. This, in turn, resulted in increased cardiac output, decreased mean dye circulation time and other evidence of improved cardiac function.

Borden, Ebert, Wilson, and Wells (10) showed that in hypertensive heart and aortic valvular disease with left ventricular dilatation, prolonged mean dye circulation time (calculated, however, by a different method) correlated in part with increased diastolic volume of the heart. Gernandt and Nylin (11) had previously demonstrated the direct relationship of prolonged circulation time to the residual volume in the dilated left ventricle. It might be inferred, therefore, that decreases in mean circulation time in patients with left ventricular dilatation are due to decreases in the diastolic volume of their hearts. However, of the nine cases in this series only one (W. A.) showed a detectable diminution of diastolic heart size roentgenographically at the time that the postoperative studies were carried out, although longer term follow-ups revealed decreased heart size in all cases (4). This apparent inconsistency can be resolved by the explanation that a steeper dye curve of shorter duration may be due not only to a decrease in the diastolic volume of the ventricle, but also to a decrease in the residual volume of the ventricle at the end of systole. If the ventricle empties more completely with more vigorous con-. traction, there will be less pooling of blood in the ventricle to delay the passage of the dye.

Newman and his colleagues (12) believe that the character of the downslope of the dye injection curves obtained in the manner described is chiefly a function of pulmonary blood volume. The alterations in the postoperative dye curve observed in our patients might thus be attributed to a decrease in the volume of the pulmonary vascular bed. However, prior to surgery, intensive medical therapy had rendered our patients as free as possible of clinical congestive heart failure. The studies of Gernandt and Nylin (11) and of Borden, Ebert, Wilson, and Wells (10) indicate that the prolonged circulation time in heart failure depends on the amount of residual blood in the heart, and only to a slight extent on the degree of congestion. Hamilton recently has criticized the validity of the calculation of pulmonary blood volume from the slopes of dye curves (13).

The concept of more complete left ventricular emptying following operation also provides an explanation for the higher systolic and lower diastolic pressures in the regurgitant brachial artery, seen after as compared to before operation. More efficient systolic contraction would by increasing stroke volume raise the systolic pressure in the 
brachial artery. More complete emptying would provide a greater pressure gradient from the regurgitant artery to the ventricle in early diastole, thereby lowering the brachial diastolic pressure.

\section{SUMMARY AND CONCLUSIONS}

1. The insertion of a plastic valvular prosthesis in the thoracic aorta, in nine patients with severe aortic insufficiency, successfully prevented regurgitant blood flow below the prosthesis.

2. In most instances, this resulted in improved cardiac function as evidenced chiefly by an increase in cardiac output and effective stroke volume, and a decrease in mean dye circulation time.

3. On the basis of the observed data, a possible sequence of events has been suggested as a mechanism by which more effective left ventricular contraction results from reduction of the absolute volume of regurgitation.

\section{REFERENCES}

1. Hufnagel, C. A., Aortic plastic valvular prosthesis. Bull., Georgetown Univ. M. Center, 1951, 4, 128.

2. Green, H. D., Circulatory dynamics. Medical Physics. Edited by Otto Glasser, Vol. II, Chicago, Yearbook Publishers, 1950, 244.

3. Hufnagel, C. A., Harvey, W. P., Rabile, P. J., and McDermott, T. F., Surgical correction of aortic insufficiency. Surgery, In press.

4. Hufnagel, C. A., Harvey, W. P., and Rabile, P. J., The clinical aspects and surgical treatment of aortic insufficiency: results of first 50 cases. In preparation.

5. Freis, E. D., Schnaper, H. W., Johnson, R. L., and Schreiner, G. E., Hemodynamic alterations in acute myocardial infarction. I. Cardiac output, mean arterial pressure, total peripheral resistance, "central" and total blood volumes, venous pressure and average circulation time. J. Clin. Invest., 1952, 31, 131.

6. Hickam, J. B., and Frayser, R., Spectrophotometric determination of blood oxygen. J. Biol. Chem., 1949, 180, 457.

7. Werkö, L., Lagerlöf, H., Bucht, H., Wehle, B., and Holmgren, A., Comparison of the Fick and Hamilton methods for the determination of cardiac output in man. Scandinav. J. Clin. \& Lab. Invest., 1949, 1, 109.

8. Hamilton, W. F., Moore, J. W., Kinsman, J. M., and Spurling, R. G., Studies on the circulation. IV. Further analysis of the injection method, and of changes in hemodynamics under physiological and pathological conditions. Am. J. Physiol., 1932, 99, 534.
9. Gibson, J. G., 2nd, and Evans, W. A., Jr., Clinical studies of the blood volume. I. Clinical application of a method employing the azo dye "Evans Blue" and the spectrophotometer. J. Clin. Invest., 1937, 16, 301.

10. Borden, C. W., Ebert, R. V., Wilson, R. H., and Wells, H. S., Studies of the pulmonary circulation. II. The circulation time from the pulmonary artery to the femoral artery and the quantity of blood in the lungs in patients with mitral stenosis and in patients with left ventricular failure. J. Clin. Invest., 1949, 28, 1138.

11. Gernandt, B., and Nylin, G., The relation between circulation time and the amount of the residual blood of the heart. Am. Heart J., 1946, 32, 411.

12. Newman, E. V., Merrell, M., Genecin, A., Monge, C., Milnor, W. R., and McKeever, W. P., The dye dilution method for describing the central circulation. An analysis of factors shaping the time-concentration curves. Circulation, 1951, 4, 735.

13. Hamilton, W. F., The physiology of the cardiac output. Circulation, 1953, 8, 527.

\section{APPENDIX}

\section{Brief Case Summaries}

1. M. H., a 31 year old colored female, had acute rheumatic fever at age 5. At age 20 she experienced the onset of progressive dyspnea on exertion, orthopnea and paroxysmal nocturnal dyspnea for which she was digitalized. Two years prior to admission she developed angina pectoris which became progressively severe. There was marked enlargement of the left ventricle. She had the murmur of free aortic regurgitation with an Austin-Flint murmur and a ventricular diastolic gallop rhythm.

On September 11, 1952, she had a plastic valvular prosthesis inserted in the thoracic aorta, and a left thoracic sympathectomy. The postoperative course was uneventful and by two weeks following surgery there was a marked diminution of the intensity of the aortic diastolic murmur, the Austin-Flint murmur and the ventricular gallop rhythm. Thirteen months after operation she was well and working full time. She was taking no digitalis, but her requirements for nitroglycerin had not significantly decreased.

2. W. A. was a 50 year old white male with central nervous system and cardiovascular syphilis. Five years before operation he developed exertional angina, and three years later progressive and incapacitating dyspnea on exertion and ankle edema. He was treated with digitalis and frequent mercurial injections. In preparation for surgery, a diuresis of approximately 40 pounds was necessary. There was marked left ventricular enlargement. $\mathrm{He}$ had the murmur of aortic insufficiency, a prominent Austin-Flint murmur, and a loud ventricular diastolic gallop. His liver was palpable but there was no peripheral edema in the immediate preoperative period.

A plastic ball valve was inserted in the descending thoracic aorta on December 2, 1952, and a left thoracic sym- 
pathectomy was performed. Postoperatively, the only complication was a transient azotemia with aggravation of previously existing paranoid tendencies. One month following operation there was a demonstrable decrease in heart size. Five months following operation he was completely asymptomatic and not taking digitalis. He did not adhere to his prescribed medical regimen. Nine months following operation he had paroxysmal auricular fibrillation, lapsed into frank congestive heart failure and developed marked azotemia. He died suddenly and autopsy disclosed multiple infarcts of mesentery, kidneys and lungs, and pyelonephritis. The plastic valve was intact.

3. C. F. was a 35 year old white male who had acute rheumatic fever at age 16. Three years prior to surgery he had auricular fibrillation converted with quinidine. Because of two subsequent attacks associated with marked dyspnea, he was placed on maintenance digitalis and quinidine. He had marked left ventricular hypertrophy. There was a murmur of free aortic insufficiency, an Austin-Flint murmur, and a ventricular diastolic gallop. Rhythm was normal sinus.

On January 12, 1953, he had a plastic valvular prosthesis inserted in the thoracic aorta. There were no postoperative complications. Three months following surgery he was working full-time, and there was a decrease in heart size on $x$-ray.

4. H. S., a 32 year old white male, had the diagnosis of aortic insufficiency of rheumatic origin made in 1945. There was no history of rheumatic fever or equivalents, and because of the presence of Marfan's syndrome, a congenital bicuspid valve damaged by endocarditis was also suspected. In 1948, he had subacute bacterial endocarditis, treated with penicillin. Three years prior to surgery, he experienced the onset of angina on exertion relieved by nitroglycerin. One year prior to admission he was digitalized and placed on salt restriction because of dyspnea on exertion and marked progression of his angina. Prior to surgery he was totally incapacitated by 20 to 30 anginal episodes daily precipitated by exertion or mental activity and often occurring at night. There were physical findings of free aortic regurgitation. The lungs were clear and there was no hepatomegaly or peripheral edema. Roentgenographically there was marked enlargement of the left ventricle.

A plastic valvular prosthesis was inserted in the thoracic aorta on February 6, 1953. Left thoracic sympathectomy was also performed. Although his postoperative course was complicated by a persistent pleural effusion, one month postoperatively there was a marked decrease in the patient's nitroglycerin requirements and a decrease in the intensity of his aortic diastolic murmur. At the time no decrease in the heart size was demonstrable by $\mathrm{x}$-ray examination. He was well and working, requiring no nitroglycerin, when he suddenly expired nine months after operation. The valve was intact but other autopsy findings are not known at this time.

5. S. E., a 52 year old white male, had acute rheumatic fever at age 11 . One and $1 / 2$ years prior to operation he noted progressive dyspnea, on exertion, orthopnea and pal- pitation. He was treated with quinidine for a tachycardia the nature of which is unknown. He was digitalized but had taken digitalis only sporadically before entrance into the hospital for surgery. He had been unable to work for one year. There was no history of angina or ankle edema. He had the murmur of free aortic regurgitation, with an Austin-Flint murmur and a ventricular diastolic gallop rhythm. In addition, there was a faint systolic murmur in the apical region which was thought to possibly represent a mild degree of mitral insufficiency. The liver was palpable two centimeters below the right costal margin and nontender. There was no edema. A marked degree of left ventricular enlargement was evident by $x$-ray examination.

A valvular prosthesis was inserted in the thoracic aorta on March 30,1953, and a left thoracic sympathectomy was performed. The postoperative course was uneventful, and by one month a definite decrease had occurred in heart size by $x$-ray examination. In two months the patient was taking no digitalis, and there was a marked diminution in both his aortic diastolic and Austin-Flint murmurs. The ventricular gallop was present but diminished. Nine months after operation he was working and apparently well.

6. L. F. was a 46 year old white male, the etiology of whose heart disease was in doubt. There was no history of rheumatic fever or equivalents. Two years prior to admission lues was diagnosed and treated with penicillin. One year prior to admission he noted the onset of progressive dyspnea on exertion and angina pectoris, requiring eventually 8 to 10 nitroglycerin tablets daily. He was not digitalized until one week prior to admission, at which time there was marked enlargement of the left ventricle and a slight degree of pulmonary congestion on the chest film. There were occasional premature ventricular contractions. Physical examination revealed the murmur of free aortic regurgitation with an Austin-Flint murmur and a ventricular diastolic gallop. There was no peripheral edema.

He had plastic aortic valvuloplasty on April 1, 1953, with left dorsal sympathectomy. Two weeks following operation the murmur of aortic insufficiency was less intense, as was the ventricular gallop, and the Austin-Flint murmur had disappeared. Twenty-five days postoperatively there was a detectable diminution in the heart size by $x$-ray examination. Nine months after operation he was working and apparently well.

7. W. J. was a 26 year old colored male who had acute rheumatic fever at age 13, with recurrence at age 14 . For one year prior to operation he had had progressive dyspnea on exertion and occasional severe precordial pain for which he had not received nitroglycerin. $\mathrm{He}$ was digitalized prior to operation. There was the murmur of free aortic regurgitation with an Austin-Flint murmur and a ventricular diastolic gallop rhythm.

On April 13, 1953, he had a plastic aortic valvuloplasty and left thoracic sympathectomy. One month following operation the Austin-Flint murmur and the gallop rhythm had completely disappeared. The aortic diastolic murmur 
had decreased several grades in intensity. Three months postoperatively he was on digitalis and was free of dyspnea and chest pain. There was a demonstrable decrease in heart size roentgenographically. Eight months following operation he died during the induction of spinal anesthesia for what was thought to be a surgical abdomen. Autopsy revealed acute gastroenteritis. The cardiovascular system was intact.

8. G. P. was a 21 year old colored male who had acute rheumatic fever in 1952 . Just prior to his bout of acute rheumatic fever he had been rejected for the draft because of a heart murmur. One year prior to operation he had progressive dyspnea on exertion and an $\mathrm{x}$-ray showed marked cardiomegaly. At that time he was digitalized. In addition to the murmur of severe aortic insufficiency and an Austin-Flint murmur, he had an aortic systolic murmur.

He had a plastic valvuloplasty on April 23, 1953, and a left thoracic sympathectomy. There were no postoperative complications. Two months postoperatively he was completely asymptomatic. There were no changes in the auscultatory findings in the heart, but there was a demonstrable decrease in heart size by $x$-ray examination. Four months following operation he died suddenly. It is known that at the time of death he was severely dyspneic and the valve was heard clicking at a rapid rate. No postmortem examination was obtained.
9. T. D. was a 40 year old colored male with a history of antiluetic therapy in 1939 . In 1952 he noted rapidly progressive dyspnea with paroxysms at night. Six months prior to surgery there were two episodes of acute pulmonary edema. At the time of operation he had been digitalized for one year, and had required many injections of mercurial diuretics. He had a loud "cooing dove" type of aortic diastolic murmur, a normal sinus rhythm, and marked cardiomegaly with the roentgenographic appearance of aneurysmal dilatation of the left ventricle.

On April 17, 1953, a plastic valve was inserted in his thoracic aorta which was markedly thickened. A left thoracic sympathectomy was also performed. There were no postoperative complications. Although there has been no essential change in the aneurysmal configuration of the heart by $x$-ray, nine months following operation the patient was on a regular diet, receiving no digitalis or diuretics, and there were no signs or symptoms of congestive heart failure. He has been noted to have occasional bouts of frequent premature ventricular contractions which have not been treated.

\section{ACKNOWLEDGMENTS}

The authors wish to acknowledge the valuable technical assistance of Miss Jean Pietras. We are indebted to Messrs. Vic Landi and Larry Gaskins for the photographic reproduction of illustrations and charts. 